\title{
Transcriptomic response of yeast cells to ATX1 deletion under different copper levels
}

\author{
Ayca Cankorur-Cetinkaya ${ }^{1,2^{*}}$, Serpil Eraslan ${ }^{1,3}$ and Betul Kirdar $^{1}$
}

\begin{abstract}
Background: Iron and copper homeostatic pathways are tightly linked since copper is required as a cofactor for high affinity iron transport. Atx1p plays an important role in the intracellular copper transport as a copper chaperone transferring copper from the transporters to Ccc2p for its subsequent insertion into Fet3p, which is required for high affinity iron transport.

Results: In this study, genome-wide transcriptional landscape of ATX1 deletants grown in media either lacking copper or having excess copper was investigated. ATX1 deletants were allowed to recover full respiratory capacity in the presence of excess copper in growth environment. The present study revealed that iron ion homeostasis was not significantly affected by the absence of ATX1 either at the transcriptional or metabolic levels, suggesting other possible roles for Atx $1 \mathrm{p}$ in addition to its function as a chaperone in copper-dependent iron absorption. The analysis of the transcriptomic response of atx1 $\Delta a t \times 1 \Delta$ and its integration with the genetic interaction network highlighted for the first time, the possible role of ATX1 in cell cycle regulation, likewise its mammalian counterpart ATOX1, which was reported to play an important role in the copper-stimulated proliferation of non-small lung cancer cells.
\end{abstract}

Conclusions: The present finding revealed the dispensability of Atx $1 p$ for the transfer of copper ions to Ccc $2 p$ and highlighted its possible role in the cell cycle regulation. The results also showed the potential of Saccharomyces cerevisiae as a model organism in studying the capacity of ATOX1 as a therapeutic target for lung cancer therapy.

Keywords: Copper transport, Copper homeostasis, Cell cycle regulation, Wilson disease, Menkes disease, Yeast, Copper chaperon

\section{Background}

Copper plays an important role in the oxidation and reduction reactions with its ability to accept and donate electrons. This ability also makes the maintenance of the homeostasis of this metal vital since it can be toxic to cells when present in excess, by inducing the formation of toxic reactive oxygen species. Thus, organisms contain various metal homeostasis factors to control the cellular accumulation, distribution, and sequestration of the metal, which is evolutionarily conserved [1]. This makes Saccharomyces cerevisiae an ideal model organism to investigate the

\footnotetext{
* Correspondence: ayca.cankorur@boun.edu.tr

'Department of Chemical Engineering, Faculty of Engineering, Bogazici University, 34342 Istanbul, Turkey

${ }^{2}$ Present address: Cambridge Systems Biology Centre \& Department of Biochemistry, University of Cambridge, CB2 1GA Cambridge, United Kingdom Full list of author information is available at the end of the article
}

copper metabolism in relation with the iron and oxygen radical metabolisms which show high overlap.

Copper is required for the oxidase activity of Fet3p [2]. The requirement of copper for trafficking of Fet3p/Ftr1p complex to the plasma membrane under iron deprived conditions represents the importance of copper for the maintenance of iron homeostasis in yeast [3]. The copper delivery pathway that mediates the transport of copper to Fet3p includes Ctr1p, Atx1p and Ccc2p [4]. Ctr1p is the high affinity copper transporter, which transfers the reduced copper by membrane reductases; Fre1p and Fre2p. The expression of CTR1 is regulated by copper level through Mac1p. It is induced under low copper conditions and repressed by high copper levels to reduce copper uptake [3]. Ctr1p is also involved in the transport of copper ions to secretory pathway. Copper is transported to cytosolic copper chaperone Atx1p, which delivers copper to Ccc2p. Atx1p was first identified as multi-copy suppressor of oxygen toxicity in 
superoxide dismutase (SOD) deficient cells [5]. However, this observation was shown to be not valid when the cells were treated with copper chelator. The decreased resistance to oxygen under copper deprived conditions were reversed by addition of copper. Copper dependent bypass of SOD deficiency and also the presence of copper ion binding domains in Atx1p indicated that this protein is involved in the intracellular transport and sequestration of copper [5].

ATX1 deleted cell exhibited no growth on iron deficient medium similar to the CTR1 and CCC2 deletion mutant, which are defective in high affinity iron uptake. Measurement of ferrous iron uptake of ATX1 deleted cells also showed a reduction, which could be restored by copper supplementation. These results indicated that Atx1p operates in the same way as Ctr1p and Ccc2p to deliver copper to Fet3p. Studies also showed that overexpression of $\mathrm{CCC} 2$ can suppress the effect of absence of ATX1 gene but the overexpression of ATX1 gene did not rescue the iron deficiency in $\mathrm{CCC} 2$ deleted strains. This finding revealed that Ccc2p functions downstream of Atx1p and there is an Atx1p-independent pathway that can reverse the effect of absence of $A T X 1$ when $C C C 2$ is overexpressed. Observation of a more severe growth defect in the temperature sensitive double deletion mutant of ATX1 and END3, absence of which blocks endocytosis, on iron deficient medium indicated that endocytosis might be the Atx1p-independent pathway that delivers copper to intracellular locations [4].

ATOX1 is the human ortholog of ATX1 and has a similar function in human. Atox1p directly interacts with the proteins encoded by $A T P 7 A$ and $A T P 7 B$, which are the Menkes and Wilson disease causing genes and delivers copper to these proteins [6]. Studies performed with Atox1 deficient mice cells demonstrated that absence of ATOX1 leads to copper accumulation due to impaired copper efflux [7]. Although no disease mutations have been reported in ATOX1 [8], Menkes disease like phenotype was observed in ATOX1 knockout mice [7].

This study concerns with the transcriptional response of the yeast cells to the absence ATX1 gene, which is the yeast ortholog of $A T O X 1$ gene in human, under different amounts of copper containing conditions. Atx1p and Ccc2p are reported to be two consecutive proteins in the intracellular transport of copper. The presence of a parallel copper trafficking pathway through endocytosis was proposed by genetic analysis but the exact mechanism still remains elusive (4). The aim of the present study was to investigate the genome-wide effects of the absence of ATX1 gene under conditions lacking copper and containing high levels of copper. The analyses of the significantly expressed genes in response to the deletion of ATX1 gene under changing copper conditions in comparison to the reference strain showed that the absence of ATX1 gene resulted in a different transcriptional re-organization than that of $C C C 2$ deletion supporting the presence of an Atx1p independent pathway that delivers copper to Ccc2p. Analysis of the transcriptome data and also further integrative analyses with genetic interaction and regulatory network highlighted a potential role of ATX1 gene in the cell cycle regulation as its mammalian counterparts.

\section{Methods}

\section{Strains and growth media}

Homozygous atx $1 \Delta / a t x 1 \Delta$ strains of $S$. cerevisiae from a genetic background of BY4743 (MATa/MATA his $3 \Delta 1$ l his $3 \Delta 1$ leu $2 \Delta 0 /$ leu $2 \Delta 0$ lys $2 \Delta 0 /+$ met $15 \Delta O /+$ ura $3 \Delta O /$ ura $3 \Delta 0$ ) were purchased from the European Saccharomyces cerevisiae Archive for Functional Analysis. Synthetic defined medium [9] without $\mathrm{CuSO}_{4}$ (copper deficient condition) and with $0.5 \mathrm{mM} \mathrm{CuSO}_{4}$ supplementation (high copper level) was used in the fermentations.

\section{Batch culture, sample description and microarray hybridization}

The fermentation experiments were carried out in duplicates using B-Braun Biostat B plus fermenters with $1.5 \mathrm{~L}$ working volume at two different levels of synthetic defined medium at $30{ }^{\circ} \mathrm{C}$ and an agitation rate of $800 \mathrm{rpm}$. The $\mathrm{pH}$ of the culture was controlled at 5.5 with $1 \mathrm{M}$ $\mathrm{NaOH}$ and $\mathrm{HCl}$. The fermentations were conducted under fully aerated conditions with an aeration rate of 1 vvm. RNA samples were collected at mid-exponential phase at an OD range of 0.7-0.8. The samples were immediately frozen in liquid nitrogen and stored at $-80{ }^{\circ} \mathrm{C}$ until further processing. The enzymatic lysis protocol of RNeasy mini kit (Qiagen, USA) with robotic workstation QIAcube (Qiagen, USA) was used for the isolation of RNA from the samples. RNA integrity was determined by Bioanalyzer 2100 (Agilent Technologies, USA) using RNA6000 Nanokit (Agilent Technologies, USA) as described by the manufacturer. The RIN values of the samples were between 7 and 10. NanoDrop (ND-1000, Thermo Scientific) was used for the quantitative and qualitative analyses of RNA.

Microarray hybridization was performed as described previously [10]. Briefly, $100 \mathrm{ng}$ of total RNA was used to synthesize first-strand cDNA then to convert into a double-stranded DNA using GeneChip ${ }^{\circ}$ ' IVT Express Kit (Affymetrix Inc., U.S.A). This double stranded cDNA was used as a template for in vitro transcription and synthesis of biotin-labelled aRNA. The final product was purified and quantified using the Nanodrop spectrophotometer before fragmentation. GeneChip reagents were used for the purification and 15 fragmentation steps. Agilent 2100 Bioanalyzer was used for the fragmented aRNA evaluation. (Agilent Technologies, Germany). The 
reagents supplied in the GeneChip ${ }^{\circ}$ Hybridization, Wash, and Stain Kit were used to prepare the Affymetrix Yeast 2.0 arrays for hybridization. A total of $5 \mu \mathrm{g}$ of aRNA was loaded onto 169 format arrays and hybridized for $16 \mathrm{~h}$. The chips were then loaded into a fluidics station for washing and staining using Affymetrix Command Console $^{\ominus}$ Software (AGCC) 3.0.1 Fluidics Control Module with Mini_euk2v3. Finally, the chips were loaded onto the Affymetrix GeneChip Scanner 3000. All applications were performed as described in the Affymetrix GeneChip Expression Analysis Technical Manual.

\section{Intracellular iron determination and sample preparation}

Samples $(24 \mathrm{ml})$ were collected from the cell cultures that were grown until mid-exponential phase in the Erlenmeyer flasks under copper deficient and high copper conditions. The samples were centrifuged at $400 \mathrm{~g}$ for $3 \mathrm{~min}$ at $4{ }^{\circ} \mathrm{C}$. The cell pellets were washed with $1 \mathrm{mM} \mathrm{KCN}$ and centrifuges at $400 \mathrm{~g}$ for $3 \mathrm{~min}$. After digestion in $5 \mathrm{ml}$ of nitric acid at $100{ }^{\circ} \mathrm{C}$ for $2 \mathrm{~h}$, the samples were centrifuged at $10000 \mathrm{~g}$ for $2 \mathrm{~min}$ and diluted with $5 \mathrm{ml}$ of water. The intracellular and extracellular iron concentrations were kindly determined by Redokslab Analytical Systems Inc., Istanbul, Turkey, using iCap Q ICP-MS (Thermo Scientific Inc., USA).

\section{Microarray data acquisition and analysis}

The cell files generated by Microarray Suite v5.0 were pre-processed with dChip software [11] using the perfect match (PM)-miss match (MS) difference model to obtain the expression levels of transcripts. These values were normalized by a baseline array with a median overall intensity. The array corresponding to reference strain with high copper condition was selected as the baseline array. The presence of single, array and probe outliers was checked and no outlier chips were identified.

The statistical analyses of the microarray data were performed using MATLAB. Significantly and differentially expressed genes in response to the deletion of ATX1 gene under changing copper levels in comparison to reference strain, were identified by two-way Analyses of Variance (2-way-ANOVA) and fold change analyses, respectively. The threshold to determine the differentially expressed genes were set to 1.5 whereas significance threshold was taken as 0.05 . The significantly and differentially expressed genes were analysed in four distinct groups. The first group, G1, included the genes that were significantly expressed only in response to the deletion of ATX1 gene and that also showed a fold change above the threshold between the reference strain and deletion mutant either under conditions lacking copper or containing high levels of copper. The second group, G2, contained the genes, which were significantly expressed only in response to copper level and which were differentially expressed between copper deficient and high levels of copper containing conditions either in the reference strain or in the deletion mutant. The third group, G3, included the genes, which were significantly and differentially expressed both in response to gene deletion and change in the copper level in an additive manner. The last group, G4 were composed of the genes, which were affected from the interactive effect of gene deletion and copper level and which showed differential expression either in response to copper or gene deletion. The significantly enriched GO biological process ontology terms $(p$-value $<0.05)$ in each group were determined using GO Term Finder [12]. The microarray data for the ATX1 deleted cells has been submitted to ArrayExpress at the European Bioinformatics Institute under accession number [E-MEXP4230] in compliance with MIAME guidelines. The data for the reference strain can be found in the ArrayExpress at the European Bioinformatics Institute under accession number [E-MEXP-3927].

\section{RT-qPCR study}

The expression level of CLN3, IME1, MSA1, MSA2, PCL9, SIC1, and ACE2 were determined under copper deficient and high levels of copper containing conditions in the reference strain and in the ATX1 deleted cells.

The primers were designed using Primer3 software, $18-24$ bases in length, with a GC content between 50 to $60 \%$ and $\mathrm{T}_{\mathrm{m}}$ in the range of $55-58{ }^{\circ} \mathrm{C}$. The complete set of primer sequences were provided in Additional file 1.

The amplicon size was determined to be either between 100 and 150 or 200 and 250 base pairs long. In order to unify the initial concentration of RNA in the analyses, all samples were diluted to the same concentration $(35 \mathrm{ng} / \mu \mathrm{l})$ prior to the real-time RTqPCR application. Reverse transcription was carried out at $50{ }^{\circ} \mathrm{C}$ for $30 \mathrm{~min}$. QuantiTect RT mix (Qiagen, USA, Cat no: 204245) was used at a ratio of 0.01 total reaction volume, which was $12.5 \mu$ l. Assays were conducted in duplicates. The synthesized cDNA template was immediately allowed to proceed with the polymerase chain reaction. Qiagen QuantiTect ${ }^{\circ} \mathrm{SYBR}^{\circ}$ Green one step RT-qPCR kit was used for real-time RT-qPCR applications as described by the manufacturer (Qiagen, USA, Cat no: 204245). All kit contents are optimized and validated by the manufacturer. The PCR reactions were performed in a final reaction volume of $12.5 \mu$ l containing the final concentration of $2.5 \mathrm{mM}$ of $\mathrm{MgCl}_{2}$ and $0.5 \mathrm{mM}$ of forward and reverse primers. Plates and adhesive seals were manufactured by Bio-Rad Laboratories (Cat no: MSB1001, MLP9601). The reaction mixtures were prepared manually and the reactions were allowed to proceed in iCycler 5 instrument (Bio-Rad Laboratories). 
iCycler $^{\text {Tw }}$ iQ Optical System Software version 3.0a (Bio-Rad Laboratories) was used with PCR base line subtracted curve fit method for the measurement of quantification cycle $(\mathrm{Cq})$. Raw $\mathrm{Cq}$ values were provided in Additional file 2. The housekeeping genes that were used to normilisation were selected using the microarray data. ARF1, TDH3 and FBA1 were identifed to be the most stable three genes among the candidate houseekeeping gene set [13] and the geometric average of the $\mathrm{Cq}$ values of these genes were used for normalisation. $2_{\mathrm{T}}^{-\Delta \Delta \mathrm{C}}$ method was used to determine the fold change between the conditions for genes of interest [14].

\section{Integration of transcriptome data with genetic interaction} and regulatory network

The genetic interactions reported in the BioGrid database (Release 3.3.123) [15] were used for the construction of genetic interaction network. The network for the genes, which are responsive to the deletion of $A T X 1$, was constructed using the genetic interaction between the genes that take place either in G1, G3 or G4. The complete genetic interaction network is constructed only for the genes that exist in the transcriptome data. Cytoscape [16] was used for the visualization and removal of the duplicate edges within the constructed network.

The reported TF-protein interactions with a documented evidence in Yeastract database [17] were used for the construction of regulatory network. The transcription factors that regulate the genes in group G1, G3 and G4 were identified from the network. The number of the genes that each TF regulates in the whole network as well as in the group of G1, G3 and G4 were determined. Enrichment analysis was conducted to identify the over-represented TFs for the genes that are responsive to the deletion of ATX1 using hypergeometric distribution function. The TFs with Bonferroni corrected $p$-value $<0.0001$ were defined as significantly enriched TFs.

\section{Results}

Cytosolic copper metallochaperon; Atx1p, is known to transport copper to Ccc2p for eventual insertion into Fet3p and its deletion causes deficiency in iron absorption, which can be supressed by addition of copper. There is also previous evidence on the existence of an Atx1p independent mechanism that transports copper to Ccc2p [4] but the exact mechanism is still elusive. Previously, we have investigated the transcriptional reorganization of the $C C C 2$ deleted cells in response to different levels of copper and identified the pathways that were affected from the disturbance of the copper homeostasis due to the absence of CCC2 gene [10]. In order to provide further insight into the role of ATX1 and observe the genome-wide effect of its absence, the transcriptional re-organisation in response to the deletion of $A T X 1$ was investigated under two different copper levels. ATX1 deletion mutant was cultivated in fully controlled fermenters in duplicates in defined medium lacking copper or containing high levels of copper $(0.5 \mathrm{mM})$, which was shown to restore the decreased respiratory capacity of the ATX1 deleted strain as it was the case for CCC2 deleted cells [10] (Additional file 3). RNA samples were collected at the mid-exponential phase.

\section{Identification of significantly and differentially expressed genes}

Genome-wide transcript levels of the ATX1 deleted and the reference strain obtained under conditions containing two different levels of copper were analysed by 2-way ANOVA to identify the significantly expressed genes and by fold change analysis to identify the differentially expressed genes (Additional file 4). The genes, which show both significant and differential change, were classified into four groups (G1-G4) and further investigated (Fig. 1). This analysis revealed that 210 genes (116 down- and 94 up-regulated) showed both significant and differential expression only in response to deletion of ATX1 gene when compared to the reference strain under both copper deficient and high levels of copper containing conditions (G1). A total of 733 genes (337 down- and 396 up-regulated) were differentially and significantly transcribed, in response to high copper levels (G2). G3 consisted of 106 genes, which showed differential and significant change both in response to gene deletion and change in the copper level without a significant interaction effect. This group of genes could further be classified into four different sub-groups; 57 genes (G3.1), which were down-regulated in response to the deletion of ATX1 when compared to the reference strain under both copper levels and up-regulated in response to high copper levels in comparison to the copper deficient conditions in both strains, 17 genes (G3.2), which were down-regulated both in the ATX1 deleted cells in comparison to the reference strain under both copper levels and under high copper containing conditions in comparison to the copper deficient conditions in both strains, 22 genes (G3.3), which were up-regulated both in the ATX1 deleted cells in comparison to the reference strain under both copper levels and under high copper containing conditions in comparison to the copper deficient condition in both strains, and 10 genes (G3.4), which showed higher expression in the ATX1 deleted cells compared to the reference strain under both copper levels and showed lower expression under high copper containing conditions when compared to the copper deficient conditions in both strains. The last group 


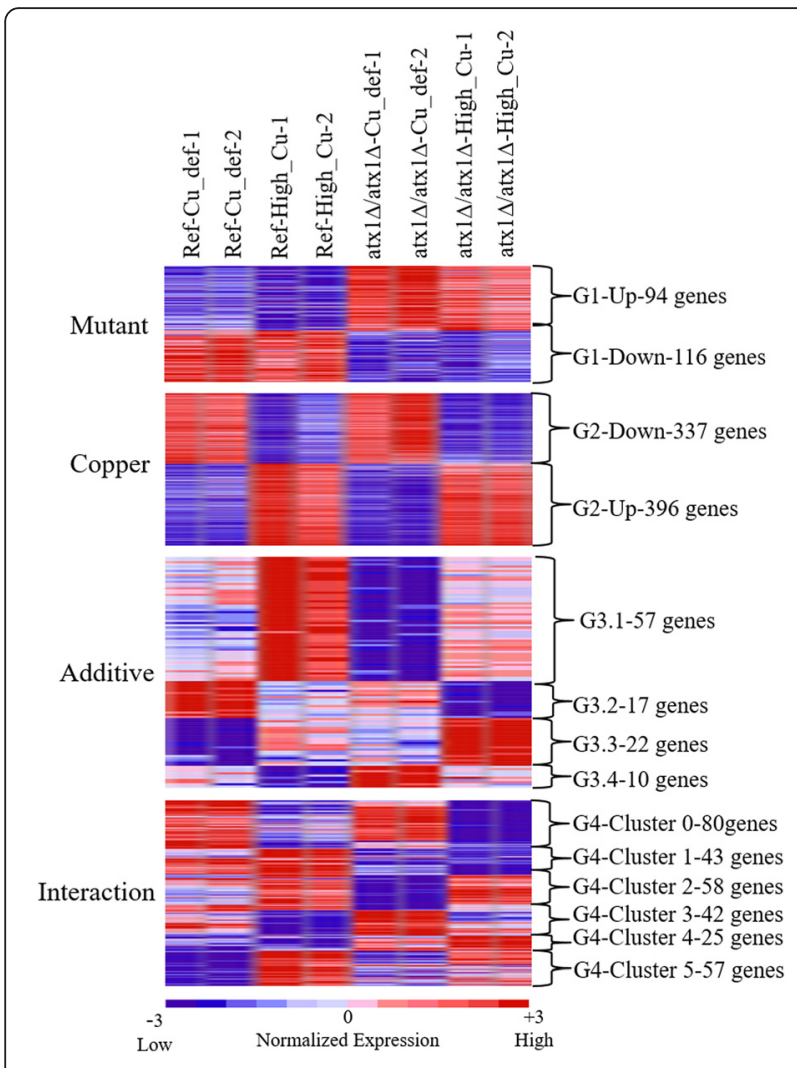

Fig. 1 Significantly and differentially expressed genes identified within each group. The figure contains the heat map representation of the genes, which were significantly and differentially expressed in response gene deletion, changing copper levels, or their additive effect, or their interactive effect. Colour key from blue to red indicates the low to high level of expression, respectively. These genes were further grouped according to the change in the direction of expression level. The number of the genes, which were identified to be up- or down-regulated in response to gene deletion effect or high copper levels were shown next to each group. Similarly, the number of the genes within each subgroup of $\mathrm{G} 3$, which compose of the genes significantly and differently expressed both in response to the gene deletion and copper level and the number of the genes within each cluster of G4, which compose of the genes significantly and differently expressed both in response to the interaction effect of gene deletion and copper level were represented next to each subgroup and cluster

of genes (G4) consisted of 305 genes displaying a significant interaction effect and differential expression either in response to changing copper levels in any strain or in response to deletion of ATX1 under any condition. The expression profiles of these genes were used to cluster these genes into 6 different clusters using Self Organizing Maps (SOM) [18] and each cluster was separately investigated. Cluster 0 composed of the genes, which showed lower level of expression under conditions containing high copper levels compared to copper deficient conditions and this response was more pronounced in the ATX1 deleted cells. Cluster 1 contained the genes, which showed lower levels of expression under conditions containing high levels of copper when compared to copper deficient conditions in the ATX1 deleted cells but higher levels of expression in the reference strain. Cluster 2 composed of the genes, which were down-regulated in the absence of ATX1 gene under copper deficient conditions in comparison to the reference strain, but not responsive under high copper containing conditions. The genes, which showed the lower level of expression in the reference strain under high levels of copper containing conditions and higher level of expression in the ATX1 deleted strain under copper deficient conditions were clustered in Cluster 3. The genes in Cluster 4 were the ones that showed higher expression under high levels of copper containing condition when compared to the copper deficient conditions in the absence of ATX1 deleted cells but not in the reference strain. Lastly, the genes that were up-regulated under copper deficient conditions but down-regulated under high levels of copper containing conditions in the ATX1 deleted cells when compared to the reference strain were clustered in Cluster 5.

\section{Effect of $A T X 1$ gene deletion}

The genes, whose expression levels were significantly $(p$-value $<0.05)$ and differentially $(\mathrm{FC}>1.5)$ changed only in response to the ATX1 deletion were identified as the transcripts that are responsive to gene deletion only (G1). This analysis revealed 116 transcripts that were significantly and differentially down-regulated in the ATX1 deleted cells when compared to the reference strain irrespective of the copper level without a significant interaction effect. These genes were identified to be significantly enriched with transcription from RNA polymerase II promoter GO biological process term $(p$-value $<0.01)$.

The manual investigation of the genes revealed downregulation of the genes involved in the cell cycle regulation in response to ATX1 deletion. The genes encoding Ime1p, which is the master regulator of meiosis, Cln3p, which is involved in the regulation of G1 to $S$ transition, Cdc39p, which is involved in cell cycle regulation, Msa1p and Msa2p, which are activators of G1 specific transcription factors involved in the regulation of G1 to $\mathrm{S}$ transition in mitotic cycle, Pog1p reported to have a putative role in the cell cycle regulation were significantly and differentially down-regulated in response to ATX1 deletion independent of the copper level. The expression levels of CLN3, IME1, MSA1 and MSA2 were further studied by real-time RT-qPCR (Additional file 5). These analyses confirmed the differential down-regulation of CLN3 under high copper level containing conditions, MSA1 under copper deficient condition and IME1 and MSA2 under both conditions in the absence of ATX1 in comparison to the reference strain. Similarly, MCM1 encoding a cell type specific transcription factor involved in 
the regulation of M/G1 and G2/M cell cycle and in the arginine catabolic process was observed to be significantly repressed in response to ATX1 deletion. There were also several genes involved in the cell cycle (VHS2, SPS1, SMC1, GIC2, PAN1, FUS3, MYO5, EGT2, CRC4, IME1, HOP2, WHI3, HRR25, SML1, SSO2, BNI5) as well as several genes encoding cell wall proteins that were found to be repressed.

A detailed manual inspection of the down-regulated genes also revealed another important group of genes (NAB3, NOP1, PIT1, NRD1, HRP1) that are involved in RNA 3' end processing. RPO21 and MED7 encoding the largest subunit and a subunit of mediator complex of RNA polymerase II, respectively were also downregulated. Targets of three down-regulated genes (SFL1, $R P H 1, R O X 1)$ were found to be significantly enriched with iron ion homeostasis and iron coordination entity transport biological process terms. $A C A 1$ encoding a TF involved in carbon source utilization, ASK1 encoding a regulator of glycerol channel, which is involved in response to oxidative stress and glycerol transport, XIR1 encoding a transcriptional repressor that regulates hypoxic genes and MIG1 encoding a TF involved in glucose repression were also observed to have significantly reduced expression levels in the absence of Atx1p. Several genes encoding proteins involved in endocytosis and ER to Golgi transport were identified among this group of genes, which are down-regulated in response to the deletion of $A T X 1$. The genes encoding a zinc transporter, Zrt3p and a zinc metalloprotease, Ste24p, a cation transporter Pmp2p and manganese ion transporter, Mam3p were also found to be negatively affected from the deletion of ATX1. The observation of the high number down-regulated genes encoding transcription factor or regulatory proteins within this group may possibly be related to a trans-regulatory function of Atx1p.

The 94 transcripts, which were up-regulated in ATX1 deleted cells, were not found to be significantly enriched with any GO biological process term. Manual inspection of this group revealed that the genes encoding proteins involved in mitochondrial fatty acid synthesis (Oar1p), inner-carnitine transporter required for carnitine dependent transport of acetyl CoA from peroxisomes to mitochondria during fatty acid beta-oxidation ( $\mathrm{Crclp}$ ), mitochondrial inter-membrane protein (Hem15p) in heme biosynthesis, mitochondrial carrier protein (Mtm1p) involved in iron homeostasis were observed to be upregulated in response to ATX1 deletion. Mtm1p is also manganese ion and pyridoxal phosphate transporter and has a role in the activation of mitochondrial Sod2p by facilitating the incorporation essential manganese cofactor. Another mitochondrial protein (Pet122p), which is a translational activator of Cox3p was also up-regulated. The genes associated with meiosis (SPO75, MSC6, MER1,
REC114 and CSM4), the genes involved in biotin (BIO3) and thiamine (THI22) biosynthesis were among the induced genes in response to deletion of ATX1 gene irrespective of the copper levels.

\section{Effect of copper level}

The genes displaying a significant $(p$-value $<0.05)$ and differential $(\mathrm{FC}>1.5)$ expression only in response to changing copper levels were identified as copper responsive genes only (G2). 337 genes were down- and 396 genes were up-regulated under high levels of copper containing conditions in comparison to the copper deficient conditions. Down-regulated genes were significantly ( $p$-value $<10^{-7}$ ) enriched with ribosome biogenesis, methylation, ncRNA processing, nitrogen compound metabolism, macromolecule methylation GO biological process terms. Up-regulated genes were found to be significantly ( $p$-value $<0.05$ ) enriched with response to stimulus and iron ion homeostasis GO biological process terms. Up-regulation of the genes encoding the high affinity iron transporter; Fet3p and siderophore iron transporters; Fth1p, Arn1p and Enb1p under high levels of copper containing conditions might indicate that high copper led to iron deficiency both in the reference and the ATX1 deleted strain. However, analyses of the intracellular and extracellular iron levels revealed that high copper levels did not result in any significant change in the intracellular and extracellular iron levels (Additional file 6). The up-regulation of iron transporters under high levels of copper containing conditions might be as a consequence of enhanced requirement for iron as a cofactor to decompose $\mathrm{H}_{2} \mathrm{O}_{2}$ in response to increased oxidative stress by high levels of copper [19].

\section{Additive effect of changing copper levels and deletion of ATX1 gene}

Inspection of 2-way ANOVA results revealed that there were also a group of genes, whose expression showed significant and differential change not only in response to deletion of ATX1 gene but also in response to changing copper levels without any significant interaction effect (G3). These genes were investigated in four different subgroups according to their responses to the perturbations.

The genes classified into G3.1 which, were downregulated in response to the deletion of ATX1 in comparison to the reference strain under both copper levels and up-regulated in response to high copper levels when compared to the copper deficient conditions in both strains, were found to be significantly $\left(p\right.$-value $<5^{*} 10^{-5}$ ) enriched with cell-wall organization and endocytosis GO biological process terms. 17 genes (G3.2), which were down- and 22 genes (G3.3), which were up-regulated, 
respectively, both in response to the deletion of $A T X 1$ gene when compared to the reference strain and in response to high copper levels in comparison to copper deficient conditions were not associated with any biological process term. The manual inspection of these groups of genes revealed that the genes encoding proteins (Cyc7p, Hug1p) involved in mitochondrial electron transport and cell cycle arrest, respectively were down-regulated. The genes (VHT1, PUT4 and MCH4), which encode transporters of $\mathrm{H}$ ${ }^{+}$-biotin, proline and monocarboxylic acid, respectively and the gene (FMP23) encoding a putative mitochondrial protein involved in iron-copper homeostasis were observed to be induced in response to ATX1 deletion under both condition and in response to high copper condition in both strains. VHT1 was previously reported to have higher mRNA levels in iron poor medium [20]. The 10 genes included into G3.4, were not identified to be significantly enriched with any process terms. 7 of these genes are not annotated with any GO Biological process term yet. YBL100W$\mathrm{A}$, and YBL005W-A, which are associated with transposition GO process term and BAT2, which encodes a cytosolic branched-chain amino acid aminotransferase were in this group.

\section{Interaction effect of changing copper levels and deletion of ATX1 gene}

The $2 \times 2$ factorial experimental design applied in this study enabled the identification of the genes, whose transcriptional responses to the deletion of ATX1 gene were copper level dependent, or the genes, with diverse transcriptional responses to changing copper levels in the ATX1 deleted and reference strains (G4).

To further classify these transcripts according to their level of expression under studied conditions, SelfOrganizing Maps (SOM) [18] was used for clustering.
These transcripts could be clustered into 6 different clusters based on their level of expression and the enriched GO biological processes among these clusters were identified (Fig. 2).

The genes in cluster 0 were down-regulated under high levels of copper containing conditions when compared to copper deficient conditions in both strains; the repression in the ATX1 deleted cells being more pronounced. These genes were significantly enriched with regulation of cell cycle and regulation of cyclin-dependent protein serine/threonine kinase activity process terms $(p$-value $<0.05)$. PCL9, which is a cyclin interacting with the Pho85 cyclin-dependent kinase that is expressed in a cell-cycle regulated manner in late M/early G1 phase of the cell cycle [21] was in this cluster. The lower expression levels of this gene under conditions containing high copper levels were also confirmed by real-time RT-qPCR analysis (Additional file 7).

The genes that showed lower expression in the ATX1 deleted strain when compared to the reference strain under both copper levels were clustered in Cluster 1. These genes were also identified to be responding high copper levels when compared to copper deficient conditions in an opposite way in two strains. These genes were not identified to be significantly enriched with any process term but manual inspection of these genes revealed the presence of the genes associated with sterol transport (NPC2, HES1, $P R Y 3)$ in this cluster. The gene encoding Sic1p, which is involved in $\mathrm{G} 1$ to $\mathrm{S}$ phase transition of mitotic cycle was also observed to be down-regulated in ATX1 deleted yeast cells under both conditions. The analysis of the expression profile of SIC1 via real-time RT-qPCR confirmed the down-regulation of this gene in the absence of $A T X 1$ under copper deficient conditions $(\mathrm{FC}=1.84$ ) (Additional file 7). Similarly SCH9

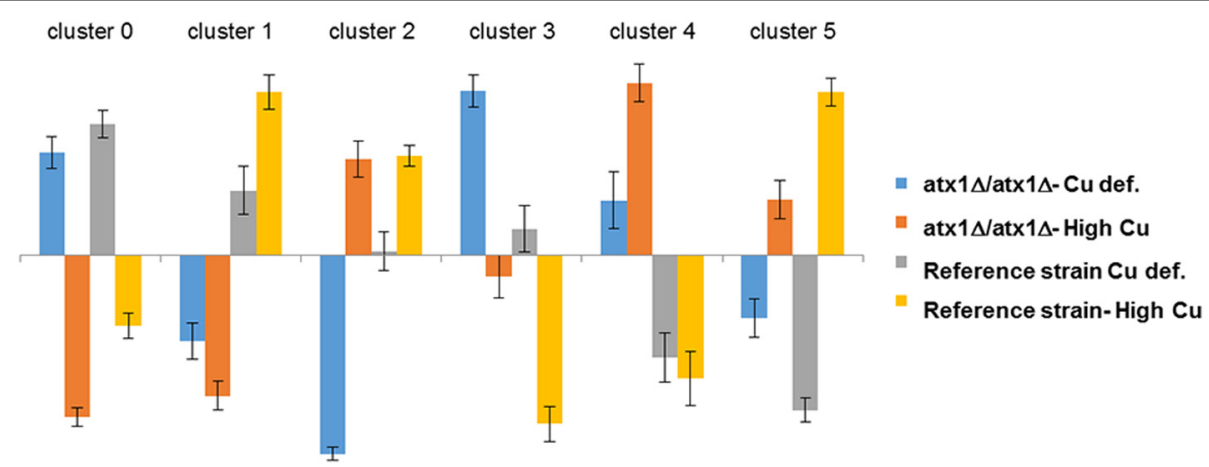

Fig. 2 Clustering of the genes which show significant interaction effect. This figure contains the average normalized expression of the genes in each cluster that are significantly and differentially expressed in response to the interacting effect of gene deletion and copper level. Error bars indicate the confidence interval around the centroids. Blue and orange represents the average level of expression in the ATX1 deleted cells under copper deficient and high copper containing conditions, respectively. Grey and yellow represents the average level of expression in the reference strain under copper deficient and high copper containing conditions, respectively 
encoding a substrate of Tor1p, which is involved in several biological processes including age dependent response to oxidative stress in chronological aging, regulation of transcription from RNA polymerase I, II and III, translation initiation and entry into G0 phase, was found to be repressed in ATX1 deleted cells. The genes encoding a zinc responsive transcription factor (Zap1p) and a membrane protein (Izh4p) involved in the zinc ion homeostasis were also found within this cluster.

The genes, which were repressed in the ATX1 deleted cells compared to the reference strain under copper deficient conditions but showed similar level of expression under high levels of copper containing conditions in these two strains, fall into Cluster 2. This cluster of genes was identified to be significantly enriched ( $p$-value $<0.05)$ with cell differentiation, cell cycle and cytokinetic cell separation process terms. $A C E 2$, which is a transcription factor that shows G2-to-M-phase-specific expression [22] was within this cluster. ACE2 was also shown to play a role in regulating the basal-level expression of CUP1 [23], encoding metallothionein that binds copper and mediates resistance to high concentrations of copper and cadmium. Further analysis of the expression level of this gene via real-time RT-qPCR showed that $A C E 2$ was respressed by 1.5 fold in the absence of ATX1 gene in comparison to the reference strain not only under copper deficient conditions but also under high copper level conditions (Additional file 7). ISU1 encoding a mitochondrial scaffold protein involved in cellular iron homeostasis and in the assembly of iron sulphur clusters and BSD2 encoding a protein involved in heavy metal ion homeostasis were also among this group of genes.

The genes that were grouped in Cluster 3 were down-regulated under high levels of copper containing conditions in comparison to the copper deficient conditions in both strains. However, these genes showed the lower level of expression in the reference strain under high levels of copper containing conditions and higher level of expression in the ATX1 deleted strain under copper deficient conditions. These genes were significantly enriched with mitotic sister chromatid cohesion and cation transport process terms ( $p$-value $<0.05)$.

Cluster 4 consists of the genes that show higher expression in the ATX1 deleted cells when compared to the reference strain under both conditions and also show higher induction in response to high copper levels when compared to copper deficient condition in the ATX1 deletion strain. A detailed manual investigation of the this group indicated that several mitochondrial genes, including $P D H 1$ encoding a protein involved in respiration, DIC1 encoding a dicarboxylic acid transporter, $Y M C 1$ encoding a putative organic acid transporter, $P K P 2$ encoding a protein kinase,
EXO5 encoding a 5'-3' exonuclease involved in mitochondrial genome maintenance, MRM2 encoding a methyl transferase required for rRNA methylation and GEM1 encoding a mitochondrial membrane GTPase are clustered into cluster 4. Gem1p is a subunit of ERMES complex that links ER to mitochondria and is involved in the inter-organelle calcium and phosphate exchange as well as mitochondrial DNA replication. The transcripts (SSA3, SPC1) associated with protein targeting to ER process term were also observed among the genes in cluster 4 .

The genes which showed lower expression under copper deficient conditions in both strains and upregulated under copper deficient conditions but down-regulated under high levels of copper containing conditions in the ATX1 deleted cells when compared to the reference strain were clustered in Cluster 5. These genes were significantly enriched with indolalkylamine catabolic process, 'de novo' NAD biosynthetic process from tryptophan, cell wall biogenesis and oxidation reduction $\mathrm{GO}$ biological process terms.

\section{Integration of genetic interaction network with transcriptional response to $A T X 1$ deletion}

Genetic interactions reflect the consequences of perturbing gene function and reveal relationships between diverse functional modules. Thus genetic interaction networks and its integration with other types of molecular data are reported to facilitate the identification of the rules governing the interdependence of genes in various cellular processes $[24,25]$. In order to provide further insight into the functional organisation of the yeast cells in response to the deletion of ATX1, a genetic interaction network was constructed using the genes that were identified to be significantly expressed in response to the deletion of ATX1 either dependent or independent of the copper levels (G1, G3 and G4). For the construction of the network, the genetic interactions in BioGrid database were used (Release 3.3.123) [15]. 430 of these genes were reported to have genetic interactions among each other yielding a connected network which contains 1223 interactions between 422 nodes (Additional file 8 ).

In order to identify the relation between $A T X 1$ and the other genes, the nodes in the network were grouped according to their distance to ATX1 (Fig. 3a). This grouping revealed that the longest path between the ATX1 and any gene in the network was 7. ATX1 has direct genetic interactions with four genes; IXR1, which encodes HMG-domain protein which binds the major DNA adducts of the antitumor drug cisplatin [26], TIR3, which encodes a cell wall mannoprotein, $R T C 2$, which encodes putative vacuolar membrane transporter for cationic amino acids, and HUR1, which encodes a protein 


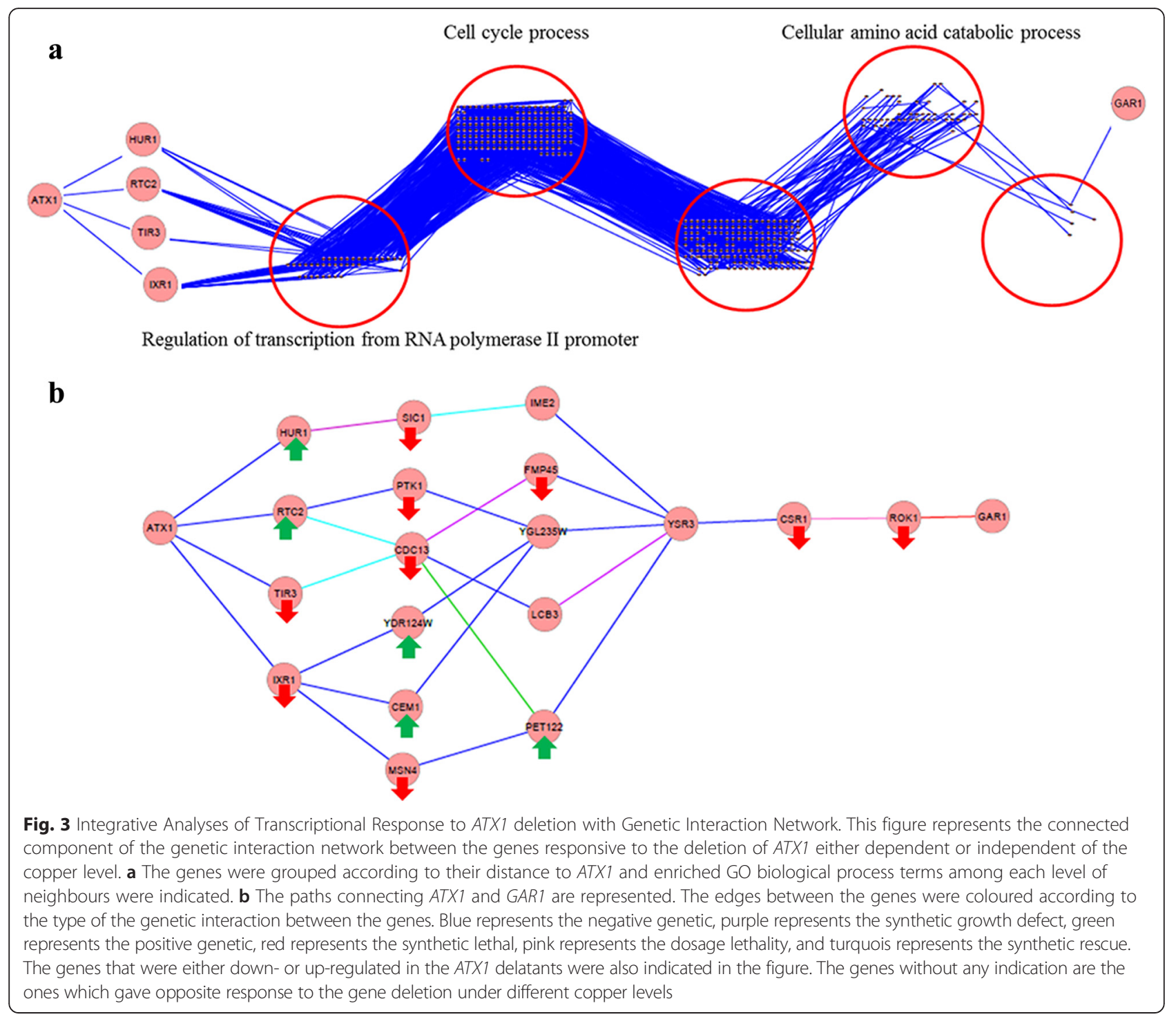

with unknown function, null mutations of which reported to show decreased metal resistance and increased ionic stress resistance [27]. 36 genes, which were second neighbours of $A T X 1$, were found to be significantly enriched with the regulation of transcription from RNA polymerase II promoter GO biological process terms. 161 genes, which were third neighbours of ATX1, were identified significantly enriched with cell cycle GO biological process term. There were 166 genes, which were the forth neighbour of ATX1 but they were not significantly enriched with any GO biological process term. There were 48 genes, which were the fifth neighbours of the ATX1 and they were significantly enriched with cellular amino acid catabolic GO biological process term. There were five genes, which were connected to ATX1 as the 6th neighbours. GAR1, which encodes a protein involved in the modification and cleavage of the $18 \mathrm{~S}$ pre-rRNA, was located at the longest distance to ATX1.
When the linear paths from ATX1 to its longest distanced neighbour GAR1 were identified (Fig. 3b), it was seen that either one of the first neighbours of the ATX1 takes place in these linear paths, whereas only 6 and 5 of the second and third neighbours fall into these paths, respectively. GAR1 was identified to be connected to third neighbour of ATX1 through ROK1, CSR1, YSR3. Investigation of these paths revealed that the genes involved in lipid biosynthesis (LCB3, CSR1, CEM1, and YSR3), cell cycle (SIC1, $I M E 2, C D C 13$, and FMP45) and rRNA processing (ROK1, GAR1) take place on the paths connecting ATX1 to GAR1. Previously, lipid metabolism and cell cycle machinery were identified to be altered in the mouse model of Wilson disease [28]. Although, we previously didn't identify a similar response in the CCC2 deleted cells, an integrative analysis the transcriptional response to the deletion of ATX1 
highlighted the alteration in the expression of the genes involved in the cell cycle and lipid biosynthesis.

When the type of the genetic interactions was investigated, it was seen that they were dominated by negative genetic interactions indicating that the mutation or deletion of the two genes at the same time results in a more severe fitness defect or lethality. The response of these genes to the deletion of ATX1 was also mapped on the graph whenever the response was not copper level dependent. However, a general trend between the type of the interaction and the direction of the change in the expression of the genes could not be observed.

A similar analysis was conducted for the complete genetic network for S. cerevisiae. This genetic interaction network was constructed for the genes, expression levels of which are studied in Yeast2 array. 5413 of those 5667 genes were reported to have genetic interactions among each other in BioGrid database (Release 3.3.123) [15], yielding a connected network with 5413 nodes and 173903 edges (Additional file 9). The nodes are grouped according to their distance to ATX1 in 4 clusters. GO enrichment analysis among the genes in each cluster revealed that the second neighbours of ATX1 were identified to be significantly enriched with cell cycle GO biological process terms. There were 2354 genes that have a connection to $A T X 1$ as a second neighbour and 417 of them were associated with cell cycle. This observation brought the question of whether enrichment of the cell cycle related genes in a network constructed only with the significantly and differentially expressed genes in response to ATX1 deletion, is significant when this complete network was considered as the background, or not. To answer this question, the significance of the existence of the 44 cell cycle related genes among the 161 third neighbours of ATX1 were calculated in a population of 2354 genes, 417 of which were associated with cell cycle using hypergeometric distribution function $(p$-value $<0.001)$. This analysis confirmed the significance of the previous finding and also provided additional evidence for the capacity of genetic interaction networks in underlying specific biological processes [24].

A similar integrative analyses were also conducted using the genes, which were reported to show a significant and differential expression in response to the deletion of $C C C 2$ gene [10]. However, since $C C C 2$ was not part of the connected network, a similar analysis could not be performed. When the nodes within the complete genetic network were grouped according to their distance to $C C C 2$, the genes were clustered into four groups and none of these groups were enriched with cell cycle related processes. First neighbours of $\mathrm{CCC} 2$ were identified to be significantly enriched with sphingolipid biosynthetic process $(p$-value $<0.01)$ and second neighbours were enriched with the terms related with vesicle-mediated transport. These findings also highlight the interdependence of $A T X 1$ and $C C C 2$ in different cellular processes.

\section{Integration of regulatory network with transcriptional response to ATX1 deletion}

An integrative approach was followed in order to elucidate the change in the landscape of the regulatory network in response to the deletion of $A T X 1$. For this purpose, the transcription factors that are responsible from the transcriptional changes in response to deletion of ATX1, were identified. To do so, the transcription factors, that are reported to be the regulators of the genes, which are members of G1, G3 and G4, were identified. In this analysis, only the interactions with a documented evidence in Yeastract database were considered. 617 of the genes in G1, G3 and G4 were identified to have reported transcription factors with documented evidence in Yeastract database. Using a similar approach to GO-term enrichment analysis, enrichment of each TF among the regulators of these 617 genes was calculated using hypergeometric distribution function. All the available TF-gene interactions with a documented evidence for all the transcripts in transcriptome data was taken as the background (Additional file 10). Twenty transcription factors were identified to be significantly enriched among 290 transcription factors (Bonferroni corrected $p$-value $<0.0001$ ). These transcription factors were Kar4p, Sok2p, Gcr2p, Yhp1p, Swi4p, Ste12p, Zap1p, Gcn4p, Tec1p, Bas1p, Hst1, Msi1, Msn2, Rap1, Sum1, Adr1, Phd1, Cup2, Fhl1, and Gzf3. Among these TFs, Msn2p, Zap1 and Gcn4p, are the regulators of the transcription from RNA polymerase II promoter in response to stress, zinc starvation and amino acid starvation respectively. Tec1p, Ste12p, Phd1p, and Sok2p are involved in the regulation of pseudohyphal growth. Kar4p regulates the genes in response to pheromones during meiotic nuclear division, Gzf3p is involved in catabolic gene expression and nitrogen catabolite repression, Rap1 is involved in chromatin silencing and in the activation of the glycolytic genes. Yhp1p, and Swi4p are transcription factors involved in mitotic cell cycle and Sum1p in negative regulation of transcription from RNA polymerase II promoter during mitosis. Fhl1p, Gcr2p are the regulators of ribosomal protein (RP) transcription. Cup2p is copper binding transcription factor and Adr1p is carbon-source responsive zinc-finger transcription factor involved in response to nutrient level. Hst1p and Msilp are transcription factors involved in the negative regulation of mitotic recombination and DNA dependent nucleosome assembly respectively. This analysis revealed the predominance of the transcription factors involved in the stress response and regulation of cell growth among the enriched TFs. 


\section{Discussion}

\section{Atx1p; a potential role in the cell cycle regulation}

Analysis of the global transcriptional response to the deletion of ATX1 gene under changing copper levels in comparison to the reference strain indicated a possible role of ATX1 gene in cell cycle related processes in Saccharomyces cerevisiae. Identification of the enriched process terms among the significantly and differentially expressed genes together with the detailed manual inspection of these genes showed that the transcripts related with cell cycle and it's transcriptional regulation were affected from the deletion of $A T X 1$ gene either irrespective of the copper level or conditionally. Absence of $A T X 1$ resulted in the repression of the genes involved in the regulation of cell cycle and induction of the genes associated with meiotic cell cycle irrespective of the copper level. The genes that play a role in the cell cycle, cell differentiation and cytokinetic cell separation processes were repressed in the ATX1 deleted cells under copper deficient conditions but not under high levels of copper containing conditions. In mammalian cells, ATOX1, which is the ortholog of ATX1, was reported to play a role in copper stimulated cyclin D1 expression and cell proliferation [29]. ATOX1 also identified to play an important role in the copperstimulated proliferation of non-small lung cancer cells and to be a potential therapeutic target for lung cancer therapy targeting copper metabolism [30]. Although ATX1 gene has not been previously associated with cell cycle in yeast, the alteration in the transcriptional response of the cell cycle associated genes to different copper levels in the absence of ATX1 gene might indicate a possible role of this protein in cell proliferation. Moreover, identification of CLN3, which is the G1 cyclin involved in cell cycle progression in yeast, to be downregulated in the $A T X 1$ deletion mutant in comparison to the reference strain under high levels of copper containing also provided additional support for this hypothesis. The down-regulation of the genes encoding Ime1p which is the master regulator of meiosis, Cdc39p which is involved in cell cycle regulation and Msa1p and Msa2p which are activators of G1 specific transcription factors in response to ATX1 deletion independent of the conditions were also supporting that Atx1p has an additional function in the yeast cells. Furthermore, integrative analysis of genetic interaction network and transcriptome revealed that IXR1, which encodes the protein binding the major DNA adducts of the antitumor drug cisplatin that is used in adjuvant chemotherapy for non-small-cell lung cancer [31], was among the first neighbours of ATX1 and downregulated in the $A T X 1$ deleted cells irrespective of the copper level in comparison to the reference strain.

Identification of the genetic interactions between the genes that are significantly expressed in response to the deletion of ATX1 also revealed that genes involved in cell cycle related process were affected from the absence of ATX1. Analysis of the network components according to their distance to ATX1 showed the genes involved in cell cycle do not have any reported direct genetic interaction with ATX1. However, the interaction between these cell cycle related genes and the ones that are involved in the regulation of transcription that are second neighbours of $A T X 1$ might indicate the possible regulatory role of $A T X 1$ in the cell cycle related processes. Furthermore, integration of the regulatory network with transcriptome also highlighted that the existence of the TFs involved in the regulation of cell growth and cell cycle related genes, among the enriched transcription factors.

The genes associated with the cell wall biogenesis and endocytosis process terms were identified to be regulated at transcriptional level both by changing copper levels and also by the deletion of the ATX1 gene. The genes involved in cell wall biogenesis were reported to be regulated in response to cell wall stress and also through cell cycle [32]. The genes associated with cell wall biogenesis process term were identified to be upregulated in response to high copper levels in comparison to copper deficient conditions in both strains and also down-regulated in the ATX1 deleted cells when compared to the reference strain under both copper levels. The deletion of ATX1 gene and changing copper levels were identified to have an interactive effect on the transcription of another group of genes associated with cell wall biogenesis. These genes were down-regulated under high levels of copper containing conditions but up-regulated under copper deficient conditions in the ATX1 deleted strain compared to the reference strain. Up-regulation of the genes involved in cell wall biogenesis in response to high copper levels can be interpreted as a response to oxidative stress [33] to mediate resistance to high copper concentrations [34]. The copper level dependent response observed in the ATX1 deleted cells may also be explained by the cell cycle dependent regulation of the genes involved in cell wall biogenesis.

It was previously shown that $\mathrm{G}_{2} / \mathrm{M}$-phase cells were more copper sensitive than $\mathrm{G}_{1} / \mathrm{S}$-phase cells and SOD1 was required for cell-cycle dependent $\mathrm{Cu}$ resistance [35]. Overexpression of the ATX1 was previously reported to substitute for $S O D 1$ by supressing the oxidative damage [36]. Recently, the molecular link between excess copper levels and the control of cell cycle and, its relation with the changes in redox balance and ROS accumulation that regulate cell proliferation was also reported in mammary epithelial cells [37]. It was shown that cells response to copper treatment by activating Cyclin D1 and $\mathrm{B} 1$ and by increasing the levels of reduced intracellular glutathione $(\mathrm{GSH})$, decreasing reactive oxygen species (ROS) generation. Copper induced Cyclin D1 expression 
was shown to be completely abolished in mouse embryonic fibroblasts lacking ATOX1 [29]. The analysis of the cell-cycle dependent $\mathrm{Cu}$ resistance in the SOD1 deleted yeast cell when ATX1 was overexpressed might give a more comprehensive picture about the link between copper resistance and cell cycle and the role of ATX1 in these processes.

\section{Atx1p and Ccc2p; two proteins with consecutive roles in copper transport but different output in their absence} Atx1p is known as a cytosolic copper metallochaperone required for copper-dependent iron absorption although a second endocytosis-dependent pathway other than Atx1p that delivers copper to Ccc2p was previously reported [4]. Yeast cells showed either decreased or complete respiratory deficiency in the absence of ATX1 and $C C C 2$ genes, respectively. This respiratory defect was shown to be recovered in the presence of high copper levels. Therefore, the transcriptional re-organisation in response to the absence of these genes was studied under copper deficient and high copper conditions to better understand the roles of these two proteins.

Previously, iron metabolism was reported to be impaired in the absence of $C C C 2$ gene under copper deficient conditions leading to iron deficiency under this condition. The transcriptional response observed in the iron transport genes in response to changing copper levels were identified to be antagonistic in the reference strain and CCC2 deleted cells [10]. On the other hand, the genes involved in the iron ion homeostasis showed a similar response to changing copper levels in the reference and ATX1 deleted cell. A decreased iron absorption in the ATX1 deleted cells was previously reported [4] but under studied conditions no significant difference was identified in the intracellular and extracellular iron levels between the ATX1 deleted cells and the reference strain. Furthermore, the genes involved in iron transport were not identified to be responsive to the deletion of ATX1 at transcriptional level. This might be explained by the incomplete blockage of iron uptake in the absence of ATX1 gene, whereas complete inhibition of transfer in the case of $C C C 2$ deletion [4]. This finding also supported the fact that an Atx1p independent pathway transfers copper to Ccc $2 p$ for further incorporation to iron transporter; Fet3p.

Endocytosis was previously proposed as a possible ATX1 independent pathway that transfers copper to Ccc2p. The analysis of the genes that are determined to be differentially and significantly expressed in this study revealed that endocytosis GO biological process term was enriched among different groups. In order to elucidate the overall change in this process, the genes that are associated with this term and also identified to be significantly and differentially expressed in this study were identified. There were 33 genes that were associated with endocytosis and showed a significant and differential change at transcriptional level. 9 of them (INP52, ART5, COS10, ARV1, PAN1, HRR25, INP53, $M Y O 5, Y P T 35)$ were responsive to the deletion of $A T X 1$ only. 10 of those (YPT53, ROG3, FTH1, MON2, ENT1, PRK1, YPK1, RIM8, AKL1, ATG20) were responsive to copper level only. There were 10 (LAS17, SCD5, ENT2, SNX41, CSR2, MYO3, SDS24, VRP1, ARK1, ROD1) and 4 (HES1, JJJ1, SVL3, SUR7) genes that were identified to be significantly and differentially expressed in response to additive and interaction effect of the perturbations, respectively. However, none of these genes were reported to have a physical interaction either with $C \operatorname{tr} 1 \mathrm{p}$ or Ccc2p.

On the other hand, up-regulation of MTM1, which encodes a mitochondrial protein that is involved in mitochondrial iron homeostasis together with the upregulation of several genes encoding mitochondrial proteins in response to deletion of ATX1, might indicate a possible disturbance in the mitochondrial iron homeostasis.

The genes involved in the NAD+ biosynthesis from tryptophan were previously identified to be affected from the absence of $C C C 2$ gene and also from the change in copper levels. The induction of BNA2 and BNA4 under copper deficient conditions in the $\mathrm{CCC} 2$ deleted cells was previously interpreted as a transcriptional reorganisation of the respiratory deficient $C C C 2$ deleted cells to maintain NAD+/NADH ratio. Previously, we have also reported that BNA2 and BNA4 were downregulated under high levels of copper containing conditions in $C C C 2$ deleted strain in contrast to the response observed in the reference strain. In the $A T X 1$ deleted cells, expression levels of $B N A 2, B N A 4$ and $B N A 1$ were identified to be induced in response to high copper levels as it was the case in the reference strain. Under copper deficient conditions, the expression level of $B N A 2$ gene was repressed more than 1.5 fold in ATX1 deleted cells when compared to the reference strain and $B N A 1$ and $B N A 4$ were expressed almost at similar levels in these two strains. The difference in this transcriptional response might be explained by the much milder decreased respiratory capacity in the ATX1 deleted cells in comparison to the $C C C 2$ deleted cells.

\section{Conclusion}

In this study, we have investigated the effect of deletion $A T X 1$ at transcriptional level under two different copper levels. This study revealed the differences between the transcriptional reorganisation in response to the absence of $\mathrm{Cu}(+2)$-transporting P-type ATPase; Ccc2p and cytosolic copper metallochaperone; Atx1p, although these proteins play consecutive functions in the intracellular 
transport of copper. This result provided additional evidence for the existence of an ATX1 independent pathway that transfers copper to Ccc2p. Furthermore, the analysis of transcriptional response to the deletion of ATX1 and also the integrative analysis of the transcriptome with genetic interaction and regulatory network revealed that the genes involved in the cell cycle regulation were significantly affected from the absence of ATX1. This finding indicated for the first time, a possible regulatory role of $A T X 1$ in cell cycle regulation, likewise its mammalian counterpart ATOX1. We believe that the observation of involvement of ATX1 in cell cycle regulation may shed light on the potential of Saccharomyces cerevisiae as a model organism to study the capacity of $A T O X 1$ as a therapeutic target for lung cancer therapy. Further investigations including analysis of transcriptomic changes in response to over-expression of ATX1 under different copper conditions will be required to enlighten the molecular mechanism of the regulatory role of Atx1p in the regulation of cell cycle. These studies will also possibly elucidate the down-regulation of the genes encoding transcriptional factors as well as that of the genes encoding proteins involved in RNA 3'-end processing, in response to the $A T X 1$ gene deletion independent of extracellular conditions.

\section{Additional files}

Additional file 1: The sequences of the reverse and forward primers of the genes of interest and the housekeeping genes. (XLSX $10 \mathrm{~kb}$ )

Additional file 2: The raw $\mathrm{Cq}$ values obtained for each gene for each sample. The values were provided as the average of the duplicate assays. (XLSX $9 \mathrm{~kb})$

Additional file 3: Spot assay to determine the respiratory capacity of the deletion mutants in comparison to reference strain. This figure represents the spot assay conducted using the reference strain, ATX1 deleted cells and CCC2 deleted cells under two different conditions; YPG, as control and $0.5 \mathrm{mM}$ copper containing YPG, to show the effect of copper supplementation on the respiratory capacity of the cells. (JPG 36 $\mathrm{kb})$

Additional file 4: The genes that were identified as significantly and differentially expressed in this study. The lists of the genes that have been identified to be significantly and differentially expressed in response to mutant effect (G1), copper effect (G2), additive effect (G3) and interaction effect (G4) are provided in the corresponding sheets together with the enriched GO process terms within in each group. (XLSX $446 \mathrm{~kb}$ )

Additional file 5: Differential expression of genes that are responsive to ATX1 deletion. The values represent the fold changes between the reference strain and the ATX1 deleted strain under corresponding conditions. Fold changes were calculated by dividing the average expression level obtained for the reference strain under corresponding condition by that of obtained for the ATX1 deletion mutant via either real-time RT-qPCR or microarray analysis. The values higher than 1.5 represent the differential down-regulation in the absence of ATX1 gene. (DOCX $12 \mathrm{~kb}$ )

Additional file 6: Intracellular and extracellular iron levels. This figure represents the intracellular (blue) and extracellular (red) iron levels in the reference and ATX1 deleted cells under copper deficient and high copper conditions. Error bars show the standard deviation. (TIF $59 \mathrm{~kb}$ )
Additional file 7: Differential expression of genes that are differentially expressed in response to interaction effect of gene deletion and copper level. The fold change values obtained between high copper and copper deficient conditions in the reference strain or ATX1 deleted cells and between ATX 1 deleted cells and the reference strain under copper deficient or high copper conditions for a) PCL9 b) S/C1 c) ACE2. Blue bars represent the fold changes calculated using the expression profiles obtained via real-time RT-qPCR and red bars represent the fold changes calculated using the expression levels obtained via microarray analysis. (TIF $189 \mathrm{~kb}$ )

Additional file 8: Genetic interaction within significant set. The genetic interaction network constructed using the genetic interactions within the genes in G1, G3 and G4 are provided in this file. The list of the nodes grouped according to their distance to ATX1 is also provided. (XLSX 778 kb)

Additional file 9: The complete genetic interaction network. The list of the nodes grouped according to their distance to ATX1 and CCC2 is provided in this file. (XLSX $6197 \mathrm{~kb})$

Additional file 10: Enriched TFs. The regulatory network and the result of the TF enrichment analysis are provided in the file. (XLSX $2909 \mathrm{~kb})$

\section{Acknowledgments}

The authors thank Dr Duygu Dikicioglu for useful discussions on network analysis.

The authors greatly acknowledge the Turkish State Planning Organization DPT09K120520, Bogazici University Research Fund through Project No 5562 and TUBITAK through Project No 110 M692 for the financial support provided for this research.

\section{Availability of data and material}

The microarray data for the ATX1 deleted cells has been submitted to ArrayExpress at the European Bioinformatics Institute under accession number [E-MEXP-4230] in compliance with MIAME guidelines. The data for the reference strain can be found in the ArrayExpress at the European Bioinformatics Institute under accession number [E-MEXP-3927].

\section{Authors' contributions}

ACC designed and performed experiments, analysed data and wrote manuscript, SE performed experiments and analysed data, BK designed experiments, analysed data and wrote manuscript. All authors read and approved the manuscript.

\section{Competing interests}

The authors declare that they have no competing interests.

\section{Author details}

${ }^{1}$ Department of Chemical Engineering, Faculty of Engineering, Bogazici University, 34342 Istanbul, Turkey. ${ }^{2}$ Present address: Cambridge Systems Biology Centre \& Department of Biochemistry, University of Cambridge, CB2 1GA Cambridge, United Kingdom. ${ }^{3}$ Present address: Diagnostic Centre for Genetic Diseases, Koc University Hospital, Davutpasa Cd. No:43010 Topkapı, Istanbul, Turkey.

Received: 5 February 2016 Accepted: 25 May 2016

Published online: 11 July 2016

References

1. Vulpe CD, Packman S. Cellular copper transport. Annu Rev Nutr. 1995;293-322.

2. Askwith C, Eide D, Van Ho A, Bernard PS, Li L, Davis-Kaplan S, Sipe DM, Kaplan J. The FET3 gene of S. cerevisiae encodes a multicopper oxidase required for ferrous iron uptake. Cell. 1994;76:403-10.

3. De Freitas J, Wintz H, Kim JH, Poynton H, Fox T, Vulpe C. Yeast, a model organism for iron and copper metabolism studies. Biometals. 2003;16:185-97.

4. Lin SJ, Pufahl RA, Dancis A, O'Halloran TV, Culotta VC. A role for the Saccharomyces cerevisiae ATX1 gene in copper trafficking and iron transport. J Biol Chem. 1997;272:9215-20.

5. Lin SJ, Culotta VC. The ATX1 gene of Saccharomyces cerevisiae encodes a small metal homeostasis factor that protects cells against reactive oxygen toxicity. Proc Natl Acad Sci U S A. 1995;92:3784-8. 
6. Liu PC, Koeller DM, Kaler SG. Genomic organization of ATOX1, a human copper chaperone. BMC Genet. 2003;4:1-4.

7. Hamza I, Faisst A, Prohaska J, Chen J, Gruss P, Gitlin JD. The metallochaperone Atox 1 plays a critical role in perinatal copper homeostasis. Proc Natl Acad Sci U S A. 2001;98:6848-52

8. Simon I, Schaefer M, Reichert J, Stremmel W. Analysis of the human Atox 1 homologue in Wilson patients. World J Gastroenterol. 2008;14:2383-7.

9. Baganz F, Hayes A, Marren D, Gardner DC, Oliver SG. Suitability of replacement markers for functional analysis studies in Saccharomyces cerevisiae. Yeast. 1997;13:1563-73.

10. Cankorur-Cetinkaya A, Eraslan S, Kirdar B. Transcriptional remodelling in response to changing copper levels in the Wilson and Menkes disease model of Saccharomyces cerevisiae. Mol Biosyst. 2013;9:2889-908.

11. Li C, Wong WH. Model-based analysis of oligonucleotide arrays: expression index computation and outlier detection. Proc Natl Acad Sci U S A. 2001;98: 31-6.

12. Boyle El, Weng S, Gollub J, Jin H, Botstein D, Michael J, Sherlock G. GO:: TermFinder-open source software for accessing Gene Ontology information and finding significantly enriched Gene Ontology terms associated with a list of genes. Bioinformatics. 2004;20:3710-5.

13. Cankorur-Cetinkaya A, Dereli E, Eraslan S, Karabekmez E, Dikicioglu D, Kirdar B. A novel strategy for selection and validation of reference genes in dynamic multidimensional experimental design in yeast. PLoS One. 2012;7:1-14.

14. Livak KJ, Schmittgen TD. Analysis of relative gene expression data using real-time quantitative PCR and the 2(-Delta Delta C(T)) Method. Methods. 2001;25:402-8.

15. Stark C, Breitkreutz BJ, Reguly T, Boucher L, Breitkreutz A, Tyers M. BioGRID: a general repository for interaction datasets. Nucleic Acids Res. 2006;34:535-9.

16. Smoot ME, Ono K, Ruscheinski J, Wang PL, Ideker T. Cytoscape 2.8: New features for data integration and network visualization. Bioinformatics. 2011; 27:431-2.

17. Abdulrehman D, Monteiro PT, Teixeira MC, Mira NP, Lourenço AB, dos Santos SC, Cabrito TR, Francisco AP, Madeira SC, Aires RS, Oliveira AL, Sá-Correia I, Freitas AT. YEASTRACT: providing a programmatic access to curated transcriptional regulatory associations in Saccharomyces cerevisiae through a web services interface. Nucleic Acids Res. 2011;39:136-40.

18. Tamayo P, Slonim D, Mesirov J, Zhu Q, Kitareewan S, Dmitrovsky E, Lander ES, Golub TR. Interpreting patterns of gene expression with self-organizing maps: methods and application to hematopoietic differentiation. Proc Natl Acad Sci U S A. 1999;96:2907-12

19. Nevitt T, Ohrvik H, Thiele DJ. Charting the travels of copper in eukaryotes from yeast to mammals. Biochim Biophys Acta. 1823;2012:1580-93.

20. Shakoury-Elizeh M, Tiedeman J, Rashford J, Ferea T, Demeter J, Garcia E, Rolfes R, Brown PO, Botstein D, Philpott CC. Transcriptional remodeling in response to iron deprivation in saccharomyces cerevisiae. Mol Biol Cell. 2004:15:1233-43.

21. Tennyson CN, Lee J, Andrews BJ. A role for the Pcl9-Pho85 cyclin-cdk complex at the M/G1 boundary in Saccharomyces cerevisiae. Mol Microbiol. 2002;28:69-79.

22. Althoefer $H$, Schleiffer A, Wassmann K, Nordheim A, Ammerer G. Mcm1 is required to coordinate G2-specific transcription in Saccharomyces cerevisiae. Mol Cell Biol. 1995;15:5917-28.

23. Butler $\mathrm{G}$, Thiele DJ. ACE2, an activator of yeast metallothionein expression which is homologous to SWI5. Mol Cell Biol. 1991;11:476-85.

24. Szappanos B, Kovács K, Szamecz B, Honti F, Costanzo M, Baryshnikova A Gelius-Dietrich G, Lercher MJ, Jelasity M, Myers CL, Andrews BJ, Boone C, Oliver SG, Pál C, Papp B. An integrated approach to characterize genetic interaction networks in yeast metabolism. Nat Genet. 2011;43:656-62.

25. Baryshnikova A, Costanzo M, Myers CL, Andrews B, Boone C. Genetic interaction networks: toward an understanding of heritability. Annu Rev Genomics Hum Genet. 2013;14:111-33.

26. McA'Nulty MM, Lippard SJ. The HMG-domain protein Ixr1 blocks excision repair of cisplatin-DNA adducts in yeast. Mutat Res - DNA Repair. 1996;362: 75-86.

27. Bleackley MR, Young BP, Loewen CJR, MacGillivray RTA. High density array screening to identify the genetic requirements for transition metal tolerance in Saccharomyces cerevisiae. Metallomics. 2011;3:195-205.

28. Huster D, Purnat TD, Burkhead JL, Ralle M, Fiehn O, Stuckert F, Olson NE, Teupser D, Lutsenko S. High copper selectively alters lipid metabolism and cell cycle machinery in the mouse model of Wilson disease. J Biol Chem. 2007;282:8343-55
29. Itoh S, Kim HW, Nakagawa O, Ozumi K, Lessner SM, Aoki H, Akram K, McKinney RD, Ushio-Fukai M, Fukai T. Novel role of antioxidant-1 (Atox1) as a copper-dependent transcription factor involved in cell proliferation. J Biol Chem. 2008:283:9157-67.

30. Cai H, Peng F. Knockdown of copper chaperone antioxidant-1 by RNA interference inhibits copper-stimulated proliferation of non-small cell lung carcinoma cells. Oncol Rep. 2013;30:269-75.

31. Scagliotti GV, Parikh P, von Pawel J, Biesma B, Vansteenkiste J, Manegold C, Serwatowski P, Gatzemeier U, Digumarti R, Zukin M, Lee JS, Mellemgaard A, Park K, Patil S, Rolski J, Goksel T, de Marinis F, Simms L, Sugarman KP, Gandara D. Phase III study comparing cisplatin plus gemcitabine with cisplatin plus pemetrexed in chemotherapy-naive patients with advancedstage Non-small-cell lung cancer. J Clin Oncol. 2008;26(21):3543-51. doi:10. 1200/JCO.2007.15.0375.

32. Levin DE. Regulation of cell wall biogenesis in saccharomyces cerevisiae: the cell wall integrity signaling pathway. Genetics. 2011;189:1145-75.

33. Smits GJ, van den Ende H, Klis FM. Differential regulation of cell wall biogenesis during growth and development in yeast. Microbiology. 2001; 147:781-94.

34. Hodgins-Davis A, Adomas AB, Warringer J, Townsend JP. Abundant geneby-environment interactions in gene expression reaction norms to copper within Saccharomyces cerevisiae. Genome Biol Evol. 2012:4:1061-79.

35. Sumner ER, Avery AM, Houghton JE, Robins RA, Avery SV. Cell cycleand age-dependent activation of Sod1p drives the formation of stress resistant cell subpopulations within clonal yeast cultures. Mol Microbiol. 2003;50:857-70.

36. Portnoy ME, Rosenzweig AC, Rae T, Huffman DL, O'Halloran TV, Culotta VC. Structure-function analyses of the ATX1 metallochaperone. J Biol Chem. 1999;274:15041-5

37. Dos Santos NV, Matias AC, Higa GSV, Kihara AH, Cerchiaro G. Copper uptake in mammary epithelial cells activates cyclins and triggers antioxidant response. Oxid Med Cell Longev. 2015;2015.

\section{Submit your next manuscript to BioMed Central and we will help you at every step:}

- We accept pre-submission inquiries

- Our selector tool helps you to find the most relevant journal

- We provide round the clock customer support

- Convenient online submission

- Thorough peer review

- Inclusion in PubMed and all major indexing services

- Maximum visibility for your research

Submit your manuscript at www.biomedcentral.com/submit
) Biomed Central 